PROCEEDINGS OF THE

AMERICAN MATHEMATICAL SOCIETY

Volume 128, Number 11, Pages 3453-3460

S 0002-9939(00)05416-2

Article electronically published on May 18, 2000

\title{
THE HOMOTOPY TYPE OF HYPERBOLIC MONOPOLE ORBIT SPACES
}

\author{
URSULA GRITSCH
}

(Communicated by Ronald A. Fintushel)

\begin{abstract}
We prove that the space $\mathcal{B}_{U(1)}^{0}$ of equivalence classes of $U(1)$ invariant connections on some $S U(2)$-principle bundles over $S^{4}$ is weakly homotopy equivalent to a component of the second loop space $\Omega^{2}\left(S^{2}\right)$.
\end{abstract}

\section{INTRODUCTION}

In this note we prove that the space $\mathcal{B}_{U(1)}^{0}$ of gauge equivalence classes of $U(1)$ invariant connections on an $S U(2)$-principle bundle over the 4-sphere $S^{4}$ of even Chern class is weakly homotopy equivalent to one component of the second loop space of the sphere $S^{2}$. In other words we prove:

Theorem 3.2.1.

$$
\mathcal{B}_{U(1)}^{0} \simeq_{\mathrm{w}} \Omega_{k}^{2} S^{2} .
$$

This $U(1)$-action on $S^{4} \subset \mathbb{R}^{5}$ is just the rotation in the $\left\langle x_{1}, x_{2}\right\rangle$-plane leaving the $x_{3}, x_{4}$ and $x_{5}$-coordinates fixed.

We use an equivariant version (see [G] theorem 2.3.1]) of a theorem in $\mathrm{AB}$. prop. 240, p.540] or [DK prop. 5.1.4, p.174], which identifies the invariant orbit space $\mathcal{B}_{U(1)}^{0}$ as an equivariant mapping space. We then analyze this mapping space using methods from equivariant homotopy theory. Usually theorems of this kind are proved using tools from analysis and gauge theory.

Anti-self-dual connections invariant under this $U(1)$-action on the 4-sphere $S^{4}$ are called hyperbolic monopoles and were first studied by Atiyah in $[\mathrm{A}$. For this reason we call the space $\mathcal{B}_{U(1)}^{0}$ the hyperbolic monopole orbit space.

In [A section 2, p.3] Atiyah shows that anti-self-dual connections on the 4-sphere $S^{4}$ invariant under this particular $U(1)$-action are in one-to-one correspondence with pairs $(A, \Phi)$, where $A$ is a connection on the trivial $S U(2)$-bundle over the hyperbolic 3 -space $\mathcal{H}^{3}$, the Higgs field $\Phi$ is a section of the adjoint bundle $\mathcal{H}^{3} \times$

Received by the editors October 30, 1998 and, in revised form, January 15, 1999

1991 Mathematics Subject Classification. Primary 58B05, 55P91.

Key words and phrases. Monopoles, gauge theory, equivariant homotopy theory.

This note is part of the author's Ph.D. thesis written at Stanford University, 1997. The author thanks her advisor Ralph Cohen for constant support and encouragement and the Studienstifung des deutschen Volkes for a dissertation fellowship. Part of this paper was written while the author was supported by an EPSRC Assistantship. 
$\mathfrak{s u}(2) \rightarrow \mathcal{H}^{3}$, and the pair $(A, \Phi)$ satisfies the Bogomolny equation $D \Phi=* F_{A}$ on the space $\mathcal{H}^{3}$ and has finite energy.

In [T] Taubes investigates the Euclidean analogue. He studies pairs $(A, \Phi)$ where $A$ is a connection on the trivial $S U(2)$-bundle over $\mathbb{R}^{3}$, the Higgs field $\Phi$ is a section of the adjoint bundle $\mathbb{R}^{3} \times \mathfrak{s u}(2) \rightarrow \mathbb{R}^{3}$ satisfying a regularity condition and the pair $(A, \Phi)$ has finite energy with respect to the Yang-Mills-Higgs functional. In $[\mathrm{T}$. Theorem A1.1, p.347] he shows that the configuration space $\mathcal{B}$ (that is the space of smooth pairs $(A, \Phi)$, where $A$ and $\Phi$ are as above) modulo the action of the based gauge group of the trivial $S U(2)$-bundle over $\mathbb{R}^{3}$ is homotopy equivalent to the space of smooth maps $f: S^{2} \rightarrow S^{2}$. The space $\mathcal{B}$ fibers over the 2 -sphere $S^{2}$ and C.H. Taubes shows that the fiber $\hat{\mathcal{B}}$ over the north pole is homotopy equivalent to the space of based smooth maps $f: S^{2} \rightarrow S^{2}$.

\section{The hyperbolic MONOPOle ORBIT SPACE $\mathcal{B}_{U(1)}^{0}$}

2.1 Notation and terminology. Let $S^{4}$ denote the 4 -sphere in $\mathbb{R}^{5}$ and let $\eta=$ $\left(P \rightarrow S^{4}\right)$ be an $S U(2)$-principal bundle over the manifold $S^{4}$. Denote by $\mathcal{A}$ the space of smooth connections on the bundle $\eta$. Let $\mathcal{G}$ be the group of smooth gauge transformations of this bundle. This means that elements $g \in \mathcal{G}$ are smooth automorphisms of $\eta$. Let $\mathcal{G}^{0}$ be the subgroup of the group $\mathcal{G}$ of gauge transformations whose elements are the identity over a given base point $m \in S^{4}$.

The circle $U(1)$ acts on the 4 -sphere $S^{4} \subset \mathbb{R}^{5}$ by the rotation in the $\left\langle x_{1}, x_{2}\right\rangle$-plane leaving the $x_{3}, x_{4}$ and $x_{5}$-coordinates fixed. We assume that the $U(1)$-action on the base $S^{4}$ lifts to the total space $P$ of the bundle $\eta$. It follows from [Ba, proposition $2.1, \mathrm{p} .434]$ and $\mathrm{A}$ 2.6, p.6] that this is the case if and only if the second Chern class $c_{2}(\eta)=n$ is even. In [Ba, p.430] P. Braam shows that the induced action of the non-trivial double cover $\tilde{U}(1)$ of $U(1)$ on the 4 -sphere $S^{4}$ always lifts to the bundle $\eta$ regardless of the parity of $n$.

By abuse of notation we denote by $\eta=\left(E=P \times_{S U(2)} \mathbb{C}^{2} \rightarrow S^{4}\right)$ the two dimensional complex vector bundle associated to the principle bundle via the standard representation of $S U(2)$ on the complex vector space $\mathbb{C}^{2}$. The complex vector bundle $\eta$ can be given the structure of a quaternionic line bundle using the isomorphisms $S U(2) \cong S p(1)$ and $\mathbb{C}^{2} \cong \mathbb{H}$ as $S U(2) \cong S p(1)$ left modules. (We consider the quaternions $\mathbb{H}$ as a right quaternionic vector space.)

We define the invariant gauge group $\mathcal{G}_{U(1)}$ to be the subgroup of the gauge group $\mathcal{G}$ such that each gauge transformation commutes with the action of the Lie group $U(1)$ on the total space $P$ of the bundle $\eta$. Analogously we define the based invariant gauge group $\mathcal{G}_{U(1)}^{0}$ to be the equivariant gauge transformations which are the identity over a given based orbit $\mathcal{O}_{m} \subset S^{4}$.

The groups $\mathcal{G}$ and $\mathcal{G}^{0}$ act naturally on the space of connections $\mathcal{A}$ from the right by the pull back of connections. We define the orbit space $\mathcal{B}$ to be the space $\mathcal{A} / \mathcal{G}$ of connections modulo the action of the gauge group and the based orbit space $\mathcal{B}^{0}$ to be the space $\mathcal{A} / \mathcal{G}^{0}$ of connections modulo the action of the based gauge group.

The left action of the group $U(1)$ on the bundle $\eta$ induces a right action on the space of connections $\mathcal{A}$ again by the pull back of connections. The fixed points of this action are called $U(1)$-invariant connections. We denote the space of fixed points by $\mathcal{A}_{U(1)}$. The invariant groups $\mathcal{G}_{U(1)}$ and $\mathcal{G}_{U(1)}^{0}$ act on the space of invariant connections $\mathcal{A}_{U(1)}$ as above. As in the non-equivariant setting we define 
the invariant orbit space $\mathcal{B}_{U(1)}$ to be the space $\mathcal{A}_{U(1)} / \mathcal{G}_{U(1)}$ of invariant connections modulo the invariant gauge group and the based invariant orbit space (the hyperbolic monopole orbit space) $\mathcal{B}_{U(1)}^{0}$ to be the space $\mathcal{A}_{U(1)} / \mathcal{G}_{U(1)}^{0}$ of invariant connections modulo the based invariant gauge group.

It is customary to complete spaces of connections in the $L^{2,2}$-Sobolev norm and elements of the gauge group in the $L^{3,2}$-Sobolev norm using a fixed connection on the bundle $\eta$. However for the purpose of this paper this is not necessary. So the reader can either think of smooth connections and smooth gauge transformations or of $L^{2,2}$-connections and $L^{3,2}$-gauge transformations.

2.2 The mapping space model of the hyperbolic monopole orbit space $\mathcal{B}_{U(1)}^{0}$. In this section we briefly describe the model for the hyperbolic monopole orbit space $\mathcal{B}_{U(1)}^{0}$ in terms of an equivariant mapping space given in $G$, theorem 2.3.1]. This model is a direct generalization of the model for the orbit space $\mathcal{B}^{0}$ given in $[\mathrm{DK}]$ and $\mathrm{AB}$ to the invariant situation. The results in this section hold for any action of a compact Lie group $H$ on a principle bundle $\eta=(P \rightarrow M)$ over any compact $H$-manifold $M$. However we state them only for $S U(2) \cong S p(1)$-principle bundles over $S^{4}$ and $H=U(1)$.

We first introduce a few notions from equivariant bundle theory. Let $V$ be a fixed quaternionic $U(1)$-representation. That means $V$ is a quaternionic right vector space with $U(1)$ left action. Let $G_{1}\left(V^{k}\right)$ be the Grassmannian of quaternionic lines in the vector space $V^{k}=\underbrace{V \oplus \cdots \oplus V}_{k \text { times }}$ for $k>1$. The group $U(1)$ acts naturally on the space $G_{1}\left(V^{k}\right)$ because it acts on the space $V^{k}$. $\left(G_{1}\left(V^{k}\right)\right.$ is just a quaternionic projective space with a $U(1)$ left action.)

We denote by $G_{1}\left(V^{\infty}\right)=\lim _{k \rightarrow \infty} G_{1}\left(V^{k}\right)$ the direct limit as a $U(1)$-equivariant space. Let $\gamma_{1}\left(V^{k}\right)=\left(E_{1}\left(V^{k}\right) \rightarrow G_{1}\left(V^{k}\right)\right)$ denote the canonical unitary bundle over the Grassmannian. The fiber over a line $P \in G_{1}\left(V^{k}\right)$ are the points $p \in$ $P$. The $U(1)$-action on the Grassmannian lifts naturally to a $U(1)$-action on the total space of the canonical bundle giving this bundle the structure of a $U(1)$ equivariant bundle. We take the limit $E_{1}\left(V^{\infty}\right)=\lim _{k \rightarrow \infty} E_{1}\left(V^{k}\right)$ and obtain the $U(1)$-equivariant bundle $\gamma_{1}\left(V^{\infty}\right)=\left(\pi: E_{1}\left(V^{\infty}\right) \rightarrow G_{1}\left(V^{\infty}\right)\right)$.

Definition 2.2.1. We call a $U(1)$-equivariant quaternionic vector bundle $\eta=\left(E \rightarrow S^{4}\right)$ subordinate to the representation $V$ if for every $m \in S^{4}$ the isotropy representation of the isotropy group $U(1)_{m}$ on the fiber over $m$ is equivalent to a sub-module of the $U(1)_{m}$-module $V$ induced by the given $U(1)$-module $V$.

The bundle $\gamma_{1}\left(V^{\infty}\right)=\left(\pi: E_{1}\left(V^{\infty}\right) \rightarrow G_{1}\left(V^{\infty}\right)\right)$ classifies vector bundles subordinate to the representation $V$. This is proved by Wasserman in Wa, section 2, p.132] for the case of real vector bundles and by Segal in [Se, section 1, p.131] for complex vector bundles. These proofs also hold in the quaternionic category.

Similarly if $\eta=(P \rightarrow M)$ is a $U(1)$-equivariant $S U(2) \cong S p(1)$-principle bundle such that the associated vector bundle $\eta=\left(E=P \times_{S p(1)} \mathbb{H} \rightarrow S^{4}\right)$ is subordinate to the representation $V$, then the frame bundle associated to the bundle $\gamma_{1}\left(V^{\infty}\right)=$ $\left(\pi: E_{1}\left(V^{\infty}\right) \rightarrow G_{1}\left(V^{\infty}\right)\right)$ classifies the principle bundle $\eta$.

Definition 2.2.2. We call $G_{1}\left(V^{\infty}\right)$ a classifying space for the bundle $\eta$ provided it is subordinate to the $U(1)$-representation $V$. 
Remark 2.2.3. Fix a point $e \in S^{4}$ which is fixed under the $U(1)$-action. Also we fix a trivialization of the bundle $\eta$ over the point $e$ once and for all. Then there is a point $* \in G_{1}\left(V^{\infty}\right)$ such that the bundle $(\eta, \eta / e)$ is classified by the pointed space $\left(G_{1}\left(V^{\infty}\right), *\right)$. Of course the base point $* \in G_{1}\left(V^{\infty}\right)$ also has to be a fixed point of the circle action on $G_{1}\left(V^{\infty}\right)$.

Proposition 2.2.4. Assume that the $U(1)$-equivariant $S U(2)$-principle bundle $\eta=$ $\left(P \rightarrow S^{4}\right)$ is subordinate to the $U(1)$-module $V$. Then there is a weak homotopy equivalence

$$
\mathcal{B}_{U(1)}^{0} \simeq_{\mathrm{w}} \operatorname{Map}_{U(1)}^{0}\left(S^{4}, G_{1}\left(V^{\infty}\right)\right)^{\eta}
$$

where the right hand side denotes the component of the mapping space of maps which classify the bundle $(\eta, \eta / e)$. (The mapping space is given the compact-open topology.)

The proof is given in [G, theorem 2.3.1]. It is a direct generalization to the invariant situation of the corresponding theorem in [DK, prop. 514, p.174].

\section{THE WEAK HOMOTOPY TYPE \\ OF THE HYPERBOLIC MONOPOLE ORBIT SPACE $\mathcal{B}_{U(1)}^{0}$}

3.1 Background and definitions. In this section we apply Proposition 2.2.4 to compute the weak homotopy type of the hyperbolic monopole orbit space $\mathcal{B}_{U(1)}^{0}$. Recall that the $U(1)$-action on $S^{4} \subset \mathbb{R}^{5}$ is just the rotation in the $\left\langle x_{1}, x_{2}\right\rangle$-plane, leaving the $x_{3}, x_{4}$, and $x_{5}$-coordinates fixed. The fixed point set is the two-sphere consisting of the points with $x_{1}=x_{2}=0$ and hence $x_{3}^{2}+x_{4}^{2}+x_{5}^{2}=1$.

However, to use methods from equivariant homotopy theory it is better to think of the sphere $S^{4}$ as the second suspension of a certain representation sphere. For an orthogonal representation $V$ of $U(1)$ let $S(V)$ denote the unit sphere in the representation $V$ and $S^{V}$ the 1-point compactification. Both spaces carry an induced $U(1)$-action. The 2 -sphere with the standard circle action given by rotation around the axis through the north and south pole is the unit sphere $S^{c}=S(1+c)$. Here $c$ denotes the standard representation of $U(1)$ on $\mathbb{C}$ and 1 denotes the trivial onedimensional representation. The 2 -sphere with the trivial circle action is the unit sphere $S^{2}=S(1+1+1)$.

For any space $X$ let $S^{0} * X$ denote the (unreduced) suspension of $X$. Hence $S^{0} * X=\frac{X \times \mathrm{I}}{X \times\{0\}, X \times\{1\}}$ where the space $I$ denotes the unit interval $[0,1]$ and we collapse the subspaces $X \times\{0\}$ and $X \times\{1\}$ to two different points. The space $S^{1} * X$ denotes the two fold suspension of $X$, i.e. we suspend the space $X$ twice.

If the space $X$ is acted on by the group $U(1)$, then the two-fold suspension $S^{1} * X$ is acted on naturally by $U(1)$ as well, namely: $\lambda \cdot[s, t, v]=[s, t, \lambda \cdot v]$ for elements $s, t \in I, v \in S^{2}$ and $\lambda \in U(1)$. There is a well-known homeomorphism between the 4 -sphere $S^{4}$ and the second unreduced suspension of the 2-sphere. Choosing this 2 -sphere to be $S^{c}$ induces a $U(1)$-action on $S^{4}$, i.e. $S^{4}=S^{1} * S^{c}$ as a $U(1)$-space.

Remark 3.1.1. We could also regard $S^{4}$ as the third unreduced suspension of the circle $U(1)$ where $U(1)$ acts on itself by multiplication. However this is not very useful when using Proposition 2.2.4: The action of $U(1)$ on itself has no fixed points but the mapping space in Proposition 2.2.4 is a based space. Since methods from equivariant homotopy theory when applied to based mapping spaces work well when one has fixed points, we prefer to work with the suspension of a $U(1)$-space with 
fixed points; for example the second suspension of the 2-sphere $S^{c}$. (This is used in the paragraph following (3.2.3).)

The $U(1)$-action on $S^{c}$ fixes the north and south poles $N$ and $S$, and hence the circle action on $S^{4}$ fixes the space $S^{1} *(N \cup S) \cong S^{2}$. Therefore the circle action on $S^{4}$ has a 2 -sphere inside the 4 -sphere as the fixed point set.

In order to apply Proposition 2.2.4 we need to compute the family of isotropy representations of the $U(1)$-action on the bundle $\eta$ and to determine a classifying space for the bundle $\eta$. The following analysis can be found in A p.4 and 5].

There are two types of isotropy groups. If a point $m \in S^{4}$ lies in the complement of the fixed point set, then the isotropy group $U(1)_{m}$ is trivial and hence the corresponding isotropy representation is trivial. If a point $m \in S^{4}$ lies in the fixed point set $S^{2} \subset S^{4}$, the isotropy group is the whole circle $U(1)$.

Let $E_{m}$ denote the fiber of the two dimensional complex vector bundle $\eta$ over the fixed point $m$. Since the structure group of $\eta$ is $S U(2)$ the $U(1)$-action on the vector space $E_{m}$ is conjugate to the standard two dimensional complex representation of $U(1)$ given by the matrix

$$
\left(\begin{array}{cc}
\lambda^{p} & 0 \\
0 & \lambda^{-p}
\end{array}\right)
$$

for some non-negative integer $p$ called the mass.

Let $A$ be any $U(1)$-invariant connection on the bundle $\eta$. For two different points $m_{1}$ and $m_{2}$ in the fixed point set $S^{2}$ the parallel transport induced by the connection $A$ defines an isomorphism as $U(1)$-modules of the fibers $E_{m_{1}}$ and $E_{m_{2}}$. Hence the mass $p$ is independent of the choice of the point $m$ in the fixed point set $S^{2} \subset S^{4}$.

The bundle $\eta$ restricted to the fixed point set $S^{2}$ splits into the direct sum of two complex line bundles $l_{p}$ and $l_{-p}$. The fiber of the bundle $l_{p}$ at a point $m \in S^{2}$ consists of the eigenspace with eigenvalue $\lambda^{p}$ for $\lambda \in U(1)$. Likewise the fiber of the bundle $l_{-p}$ consists of eigenspaces with eigenvalues $\lambda^{-p}$ for $\lambda \in U(1)$. The line bundles $l_{p}$ and $l_{-p}$ are complex conjugate to each other.

Definition 3.1.1. Let $k$ be the first Chern class $c_{1}\left(l_{p}\right)$ of the bundle $l_{p}$. The integer $k$ is called the charge.

It is well known [A, 2.6, p.6] that there is the equality $n=2 p k$ between the mass, charge and the second Chern class of the bundle $\eta$.

If we consider the bundle $\eta$ as a quaternionic line bundle, then over the fixed point set $S^{2}$ the isotropy representation of $U(1)$ is conjugate to the one dimensional quaternionic $U(1)$-module $\mathbb{H}$ where an element $\lambda \in U(1)$ acts by left multiplication with the element $\lambda^{p}$ on the quaternions $\mathbb{H}$. We say that as a $U(1)$-module $\mathbb{H}$ has weight $p$. Hence as a quaternionic line bundle the bundle $\eta$ is subordinate to the $U(1)$-module $\mathbb{H}$ of weight $p$ and therefore the equivariant Grassmannian $G_{1}\left(\mathbb{H}^{\infty}\right)=\mathbb{H} P^{\infty}$ classifies the bundle $\eta$.

We choose the point $e:=[1,1, N] \in S^{1} * S^{c}$ as the based orbit where $N \in S^{c}$ is the north pole. Also we fix a trivialization of the bundle $\eta$ over the point $e$ once and for all. Then, as mentioned in Remark 2.2.3, there is a point $* \in G_{1}\left(\mathbb{H}^{\infty}\right)$ such that the bundle $(\eta, \eta / e)$ is classified by the pointed space $\left(G_{1}\left(\mathbb{H}^{\infty}\right), *\right)$ for some point $* \in G_{1}\left(\mathbb{H}^{\infty}\right)$. Of course the base point $* \in G_{1}\left(\mathbb{H}^{\infty}\right)$ also has to be a fixed point of the circle action on $G_{1}\left(\mathbb{H}^{\infty}\right)$. 
3.2 Proof of the main theorem. For any topological space $X$ denote by $\Omega^{2} X=$ $\operatorname{Map}^{0}\left(S^{2}, X\right)$ the based continuous maps from the 2-sphere $S^{2}$ into $X$ together with the compact-open topology. This space has components labeled by the second homotopy group $\pi_{2}(X)$ of $X$. For some element $k \in \pi_{2}(X)$ let $\operatorname{Map}_{k}^{0}\left(S^{2}, X\right)$ denote the component labeled by $k$. Recall that the hyperbolic monopole orbit space $\mathcal{B}_{U(1)}^{0}=\mathcal{A}_{U(1)} / \mathcal{G}_{U(1)}^{0}$ is the space of $U(1)$-invariant connections on the bundle $\eta$ modulo the based equivariant gauge group $\mathcal{G}_{U(1)}^{0}$.

Theorem 3.2.1. There is a weak homotopy equivalence $\mathcal{B}_{U(1)}^{0} \simeq_{\mathrm{w}} \Omega_{k}^{2} S^{2}$, where $k$ is the charge of the bundle $\eta$ as defined in section 3.1 and $\Omega_{k}^{2} S^{2}$ denotes the component of the second loop space $\Omega^{2} S^{2}$ labeled by the charge $k$.

Proof. Proposition 2.2.4 already gives a weak homotopy equivalence

$$
\mathcal{B}_{U(1)}^{0} \simeq_{\mathrm{w}} \operatorname{Map}_{U(1)}^{0}\left(S^{4}, G_{1}\left(\mathbb{H}^{\infty}\right)\right)^{\eta} .
$$

Here the superscript $\eta$ in the mapping space denotes the component of maps which classify the bundle $\eta$. We will first show that there is a homotopy equivalence

$$
\operatorname{Map}_{U(1)}^{0}\left(S^{4}, G_{1}\left(\mathbb{H}^{\infty}\right)\right) \simeq \Omega^{2} S^{2} .
$$

In section 3.3 we will identify which components correspond to each other under this homotopy equivalence.

For two pointed topological spaces $\left(X, x_{0}\right)$ and $\left(Y, y_{0}\right)$ define the smash $X \wedge Y$ to be the pointed space $\frac{X \times Y}{X \times\left\{y_{0}\right\} \cup\left\{x_{0}\right\} \cup Y}$. If the spaces $X$ and $Y$ are acted on by the circle group $U(1)$ with fixed points $x_{0}$ and $y_{0}$, then the smash $X \wedge Y$ is acted on in a natural fashion by the circle $U(1)$ as well. Hence the circle group $U(1)$ acts on the smash $S^{2} \wedge S^{c}$ where we have chosen the north pole in both 2-spheres as the base point. Here we recall that $S^{c}$ denotes the 2 -sphere with the standard circle action and $S^{2}$ the 2-sphere with the trivial circle action.

We have identified $S^{4}=S^{1} * S^{c}$ and there is a well-known $U(1)$-equivariant homotopy equivalence $S^{1} * S^{c} \simeq S^{2} \wedge S^{c}$. Hence we have to analyze the mapping space $\operatorname{Map}_{U(1)}^{0}\left(S^{2} \wedge S^{c}, G_{1}\left(\mathbb{H}^{\infty}\right)\right)$. Taking $U(1)$-fixed points of the equivariant homeomorphism

$$
\begin{aligned}
\operatorname{Map}^{0}\left(S^{2} \wedge S^{c}, G_{1}\left(\mathbb{H}^{\infty}\right)\right) & \cong \operatorname{Map}^{0}\left(S^{2}, \operatorname{Map}^{0}\left(S^{c}, G_{1}\left(\mathbb{H}^{\infty}\right)\right)\right) \\
& \cong \Omega^{2} \operatorname{Map}^{0}\left(S^{c}, G_{1}\left(\mathbb{H}^{\infty}\right)\right)
\end{aligned}
$$

gives the homotopy equivalence

$$
\operatorname{Map}_{U(1)}^{0}\left(S^{4}, G_{1}\left(\mathbb{H}^{\infty}\right)\right) \simeq \Omega^{2} \operatorname{Map}_{U(1)}^{0}\left(S^{c}, G_{1}\left(\mathbb{H}^{\infty}\right)\right) .
$$

The proof of the homotopy equivalence (3.2.3) follows now from Lemma 3.2.5 below.

Lemma 3.2.5. There is a homotopy equivalence $\operatorname{Map}_{U(1)}^{0}\left(S^{c}, G_{1}\left(\mathbb{H}^{\infty}\right)\right) \simeq S^{2}$.

Proof. The sphere $S^{c}$ is $U(1)$-equivariantly obtained by gluing in along the north and south pole a $U(1)$-equivariant 1-cell. Hence there is a $U(1)$-equivariant cofibration sequence

$$
U(1)_{+} \rightarrow S^{0} \rightarrow S^{c}
$$

where the zero dimensional sphere $S^{0}$ consists of the north and south pole of the sphere $S^{c}$. Applying the equivariant mapping functor $\operatorname{Map}_{U(1)}^{0}\left(\ldots, G_{1}\left(\mathbb{H}^{\infty}\right)\right)$ to the 
sequence (3.2.6) gives the fibration sequence

(3.2.7)

$$
\operatorname{Map}_{U(1)}^{0}\left(U(1)_{+}, G_{1}\left(\mathbb{H}^{\infty}\right)\right) \leftarrow \operatorname{Map}_{U(1)}^{0}\left(S^{0}, G_{1}\left(\mathbb{H}^{\infty}\right)\right) \leftarrow \operatorname{Map}_{U(1)}^{0}\left(S^{c}, G_{1}\left(\mathbb{H}^{\infty}\right)\right) .
$$

We have the homeomorphisms

$$
\begin{aligned}
\operatorname{Map}_{U(1)}^{0}\left(U(1)_{+}, G_{1}\left(\mathbb{H}^{\infty}\right)\right) & \cong G_{1}\left(\mathbb{H}^{\infty}\right)=B S U(2) \\
\text { and } \operatorname{Map}_{U(1)}^{0}\left(S^{0}, G_{1}\left(\mathbb{H}^{\infty}\right)\right) & \cong G_{1}\left(\mathbb{H}^{\infty}\right)^{U(1)} .
\end{aligned}
$$

Lemma 3.2.8. There is a homeomorphism $G_{1}\left(\mathbb{H}^{\infty}\right)^{U(1)} \cong G_{1}\left(\mathbb{C}^{\infty}\right)=B U(1)$.

Hence the mapping space $\operatorname{Map}_{U(1)}^{0}\left(S^{c}, G_{1}\left(\mathbb{H}^{\infty}\right)\right)$ is the homotopy fiber of the standard map $B U(1) \rightarrow B S U(2)$ which is, up to homotopy, the space $S^{2}$. This proves Lemma 3.2.5 and finishes the proof of (3.2.3).

Proof of Lemma 3.2.8. Let $\tau: G_{1}\left(\mathbb{C}^{\infty}\right) \hookrightarrow G_{1}\left(\mathbb{H}^{\infty}\right)$ be the map induced by the natural inclusion $\mathbb{C} \hookrightarrow \mathbb{H}$. Recall that we view the space $\mathbb{H}^{\infty}$ as a right quaternionic vector space endowed with the $U(1)$-action given by left multiplication of weight $p$. Hence the image $\tau\left(G_{1}\left(\mathbb{C}^{\infty}\right)\right)$ lies in the fixed point set $G_{1}\left(\mathbb{H}^{\infty}\right)^{U(1)}$ of the induced circle action on the Grassmannian $G_{1}\left(\mathbb{H}^{\infty}\right)$ and the map $\tau$ induces a continuous $\operatorname{map} \tilde{\tau}: G_{1}\left(\mathbb{C}^{\infty}\right) \hookrightarrow G_{1}\left(\mathbb{H}^{\infty}\right)^{U(1)}$.

Let $w=\left[x_{0}, x_{1}, \ldots\right]$ be a quaternionic line in $\mathbb{H}^{\infty}$ fixed under the circle action. Without loss of generality we may assume $x_{0}=1$. Given $\lambda \in U(1)$ there is an element $\alpha(\lambda) \in S p(1)$ such that $\lambda^{p} x_{i}=x_{i} \alpha(\lambda)$ for all $i=0,1, \ldots$ For $i=0$ this gives $\alpha(\lambda)=\lambda^{p}$. Hence $\lambda^{p} x_{i}=x_{i} \lambda^{p}$ for all $i=0,1, \ldots$ and hence $x_{i}$ lies in the centralizer of $U(1)$ in the quaternions $\mathbb{H}$ which is equal to $\mathbb{C}$.

Hence $x_{i} \in \mathbb{C}$ for all $i=0,1, \ldots$ and the element $w=\left[x_{0}, x_{1}, \ldots\right]$ lies in the image of the map $\tilde{\tau}: G_{1}\left(\mathbb{C}^{\infty}\right) \rightarrow G_{1}\left(\mathbb{H}^{\infty}\right)^{U(1)}$. The map $\tilde{\tau}$ induces a homeomorphism $G_{1}\left(\mathbb{C}^{\infty}\right) \cong G_{1}\left(\mathbb{H}^{\infty}\right)^{U(1)}$. This finishes the proof of Lemma 3.2.8.

3.3 Identification of the component. We now finish the proof of Theorem 3.2.1. Recall that we denoted by $\eta=\left(P \rightarrow S^{4}\right)$ a fixed $U(1)$-equivariant principal $S U(2)$ bundle over the 4 -sphere $S^{4}$ with second Chern class $c_{2}(\eta)=n$. Restricted to the fixed point set $S^{2} \subset S^{4}$ of the $U(1)$-action on the 4-sphere the bundle $\eta$ is induced from a $U(1)$-bundle $\nu$ with first Chern class $c_{1}(\nu)=k$. In Definition 3.1.1 we defined the number $k$ to be the charge of the bundle $\eta$.

Let $\Gamma: \operatorname{Map}_{U(1)}^{0}\left(S^{4}, G_{1}\left(\mathbb{H}^{\infty}\right)\right) \rightarrow \Omega^{2} S^{2}$ be the map inducing the homotopy equivalence in (3.2.3). We want to determine to which component of the double loop space $\Omega^{2} S^{2}$ the map $\Gamma$ maps the connected component $\operatorname{Map}_{U(1)}^{0}\left(S^{4}, G_{1}\left(\mathbb{H}^{\infty}\right)\right)^{\eta}$ of the mapping space $\operatorname{Map}_{U(1)}^{0}\left(S^{4}, G_{1}\left(\mathbb{H}^{\infty}\right)\right)$. For this it is enough to compute the degree of the map $\Gamma(f): S^{2} \rightarrow S^{2}$ for one map $f \in \operatorname{Map}_{U(1)}^{0}\left(S^{4}, G_{1}\left(\mathbb{H}^{\infty}\right)\right)^{\eta}$.

We identify the 4-sphere $S^{4}$ with the smash $S^{2} \wedge S^{c}$ as before. Here the circle $U(1)$ acts trivially on the first factor and by rotation around the axis through the north and south pole on the second factor. The fixed point set of the circle action on $S^{4}$ is then the subspace $\left\{[z, S] \in S^{2} \wedge S^{c}: z \in S^{2}\right\}$ which is just isomorphic to a copy of $S^{2}$. Here $S$ denotes the south pole of the second factor $S^{c}$.

Denote by $j: S^{2} \rightarrow \operatorname{Map}_{U(1)}^{0}\left(S^{c}, G_{1}\left(\mathbb{H}^{\infty}\right)\right)$ a map inducing the homotopy equivalence from Lemma 3.2.5 and let $\iota^{*}: \operatorname{Map}_{U(1)}^{0}\left(S^{c}, G_{1}\left(\mathbb{H}^{\infty}\right)\right) \rightarrow \operatorname{Map}_{U(1)}^{0}\left(S^{0}, G_{1}\left(\mathbb{H}^{\infty}\right)\right)$ $=B U(1)$ be the map on the mapping spaces induced by the cofibration $\iota: S^{0} \hookrightarrow S^{c}$ from (3.2.6). Let $\pi=\iota^{*} \circ j: S^{2} \rightarrow B U(1)$ the composition. It is clear that the 
map $\pi$ induces the identity on second cohomology. Hence in order to calculate the degree of the map $\Gamma(f)$ it is enough to calculate the map

$$
(\pi \circ \Gamma(f))^{*}: H^{2}(B U(1), \mathbb{Z}) \rightarrow H^{2}\left(S^{2}, \mathbb{Z}\right) .
$$

By construction the map $\pi \circ \Gamma(f)$ is a lift of the classifying map $f$ of the bundle $\eta$ to the fixed point set $S^{2}$ via the map $B(\iota): B U(1) \hookrightarrow B S U(2)$. Since the bundle $\eta$ restricted to the fixed point set $S^{2} \subset S^{4}$ is induced from a $U(1)$-bundle $\nu$ with first Chern class $c_{1}(\nu)=k$, the map $\pi \circ \Gamma(f): S^{2} \rightarrow B U(1)$ is a classifying map for the bundle $\nu$ and hence the map $(\pi \circ \Gamma(f))^{*}$ in (3.3.1) is multiplication by $k$. This finally finishes the proof of Theorem 3.2.1.

\section{REFERENCES}

[A] Atiyah, M.F.: Magnetic monopoles on hyperbolic spaces. In Proc. of Bombay Colloquium 1984 on "Vector Bundles on Algebraic Varieties", 1987, 1-34, Oxford: Oxford University Press. MR 88i:32045

[AB] Atiyah, M.F., and Bott, R.: The Yang-Mills equation over Riemann surfaces, Phil. Trans. R. Soc. Lond. A 308 (1982), 523-615. MR 85k:14006

[Ba] Braam, P.J.: Magnetic Monopoles on three-manifolds, J. Differential Geometry Vol. 30 (1989), 425-464. MR 90e:53040

[DK] Donaldson, S.K., and Kronheimer, P.B.: "The Geometry of Four-Manifolds", Oxford mathematical monographs, 1990, Oxford: Oxford University Press. MR 92a:57036

[G] Gritsch, U.: Morse theory for the Yang-Mills functional via equivariant homotopy theory, 1997, preprint.

[Se] Segal, G.B.: Equivariant K-theory, Publ. Math. Inst. Hautes Études Sci. 34 (1968), 129-151. MR 38:2769

[T] Taubes, C.H.: Monopoles and Maps from $S^{2}$ to $S^{2}$, the Topology of the Configuration Space, Commun. Math. Phys. 95 (1984), 345-391. MR 86i:58032

[Wa] Wasserman, A.G.: Equivariant differential topology, Topology Vol. 8, 127-150. MR 40:3563

Department of Pure Mathematics and Mathematical Statistics, University of CamBridge, 16 Mill Lane, Cambridge, CB2 1SB, United Kingdom

E-mail address: ursula@dpmms.cam.ac.uk

Current address: Department of Mathematics, University of California at Berkeley, Evans Hall, Berkeley, California 94705

E-mail address: ursula@math.berkeley.edu 